Research Paper

\title{
A Novel Role of Cab45-G in Mediating Cell Migration in Cancer Cells
}

Judong Luo ${ }^{12^{*}}$, Zengpeng $\mathrm{Li}^{3^{*}}$, Hong Zhu ${ }^{4}$, Chenying Wang 3 , Weibin Zheng ${ }^{3}$, Yan $\mathrm{He}^{5}$, Jianyuan Song ${ }^{5}$, Wenjie Wang ${ }^{6}$, Xifa Zhou ${ }^{1}$, Xujing Lu$^{1}$, Shuyu Zhang ${ }^{5,6}{ }^{\boxplus}$, Jianming Chen ${ }^{3 凶}$

1. Department of Radiotherapy, Changzhou Tumor Hospital, Soochow University, Changzhou, 213001, China

2. Department of Radiation Oncology, Shandong Cancer Hospital affiliated to Shandong University, Shandong Academy of Medical Sciences, Jinan, 250117, China;

3. Key Laboratory Breeding Base of Marine Genetic Resources, Third Institute of Oceanography, State Oceanic Administration, Xiamen 361005, China;

4. Department of Radiation Oncology, Minhang Branch of Cancer Hospital of Fudan University, Shanghai 200240, China;

5. School of Radiation Medicine and Protection and Jiangsu Provincial Key Laboratory of Radiation Medicine and Protection, Medical College of Soochow University, Suzhou 215123, China;

6. Collaborative Innovation Center of Radiation Medicine of Jiangsu Higher Education Institutions and School for Radiological and Interdisciplinary Sciences (RAD-X), Soochow University, Suzhou 215123, China.

*These first three authors contributed equally to this work.

$\triangle$ Corresponding authors: (S. Zhang) Tel/Fax: +86512-65880065, E-mail: zhang.shuyu@hotmail.com; (J. Chen) Tel/Fax: +86592-2195337, E-mail: chenjm@xmu.edu.cn.

(C) Ivyspring International Publisher. Reproduction is permitted for personal, noncommercial use, provided that the article is in whole, unmodified, and properly cited. See http://ivyspring.com/terms for terms and conditions.

Received: 2014.11.11; Accepted: 2016.03.19; Published: 2016.04.25

\begin{abstract}
$\mathrm{Ca}^{2+}$-binding protein of $45 \mathrm{kDa}$ (Cab45), a CREC family member, is reported to be associated with $\mathrm{Ca}^{2+}$-dependent secretory pathways and involved in multiple diseases including cancers. Cab45-G, a Cab45 isoform protein, plays an important role in protein sorting and secretion at Golgi complex. However, its role in cancer cell migration remains elusive. In this study, we demonstrate that Cab45-G exhibited an increased expression in cell lines with higher metastatic potential and promoted cell migration in multiple types of cancer cells. Overexpression of Cab45-G resulted in an altered expression of the molecular mediators of epithelial-mesenchymal transition (EMT), which is a critical step in the tumor metastasis. Quantitative real-time PCR showed that overexpression of Cab45-G increased the expression of matrix metalloproteinase-2 and -7 (MMP-2 and MMP-7). Conversely, knock-down of Cab45-G reduced the expression of the above MMPs. Moreover, forced expression of Cab45-G upregulated the level of phosphorylated ERK and modulated the secretion of extracellular proteins fibronectin and fibulin. Furthermore, in human cervical and esophageal cancer tissues, the expression of Cab45-G was found to be significantly correlated with that of MMP-2, further supporting the importance of Cab45-G on regulating cancer metastasis. Taken together, these results suggest that $\mathrm{Cab} 45-\mathrm{G}$ could regulate cancer cell migration through various molecular mechanisms, which may serve as a therapeutic target for the treatment of cancers.
\end{abstract}

Key words: Cab45-G, cell migration, Golgi complex, epithelial-mesenchymal transition (EMT).

\section{Introduction}

The CREC (Cab45/reticulocalbin/ERC45/ calumenin) family proteins include $\mathrm{Ca}^{2+}$-binding protein of $45 \mathrm{kDa}$ (Cab45), reticulocalbin, ER $\mathrm{Ca}^{2+}$-binding protein of $55 \mathrm{kDa}$ (ERC-55) and calumenin, which are encoded by different genes [1]. These proteins are expressed ubiquitously with varied levels in different tissues. Almost all CREC family members consist of multiple EF-hand motifs, which show high affinity to calcium [2]; however, for CREC family proteins, the binding affinity is very low with dissociation constants up to the $\mathrm{mM}$ level [3]. Functionally, due to the various localization in endoplasmic reticulum, Golgi lumen or cell surface, the CREC family members are reported to be 
associated with $\mathrm{Ca}^{2+}$-dependent secretory pathways $[4,5]$. In addition, these proteins also function in various diseases, such as liver cancer [6], neuromuscular [7] and cardiovascular diseases [8].

The SDF4 gene, which contains seven exons and maps to $1 \mathrm{p} 36.33$ in chromosomes, encodes the 362-amino acid Cab45 protein [9]. Cab45 precursor embraces a signal sequence, six EF-hands and a HEEF motif at the C-terminal [4, 10]. Alternative splicing of Cab45 mRNA results in three members: Cab45-G [10], Cab45-C [11] and Cab45-S [12]. Cab45-G is named by its main localization to Golgi complex. The C-terminal 130 amino acid variant of Cab45-G is localized in the cytosol due to the lack of signal peptide, therefore termed Cab45-C. The third splice variant Cab45-S is secreted and differs from Cab45-G in the C-terminal sequences with no sixth EF-hand or HEEF motif [4].

The majority of cancer patients are diagnosed after tumors have widely spread, limiting the effectiveness of debulking surgery, radiotherapy and chemotherapy [13]. The presence of metastasis in regional lymph nodes is a strong indicator of poor patient survival [14]. Activation of epithelial-mesenchymal transition (EMT), a traditional phenomenon revealed in embryonic development, has been recognized important for cancer cell metastaisi. During this process, static epithelial cells lose cell to cell junctions and as a consequence they lose apico-basal polarity to become migratory mesenchymal-like cells. Epithelial features, such as cellular adhesion marker E-cadherin and intracellular adhesion components such as tight junctions, cytokeratins, and desmosomes, are downregulated. Coordinating with this downregulation is an upregulation of mesenchymal markers such as N-Cadherin, Vimentin, and Fibronectin3 [15, 16]. The key molecules driving cancer metastasis are still poorly understood and one of the biggest areas in cancer biology research is elucidating the mechanisms involved in cancer metastasis.

Several CREC family members have been implicated in cancer progression. For example, Cab45-S regulates the $\mathrm{Ca}^{2}$ level of endoplasmic reticulum and acts as a crucial modulator of tumor growth in cervical cancer cells [17]. However, the expression and function of Cab45-G in cancer progression remains elusive. Here we show that Cab45-G is involved in the migration of cancer cells through various molecular mechanisms, which may serve as a therapeutic target for the treatment of cancers.

\section{Materials and Methods}

\section{RNAi Sequences and Antibodies}

Two Cab45-G shRNA vectors, named shCab45G-1 (GGTTCATGGTGAAGGAGAT) and shCab45G-2 (GGAAGCTGATGGTCATCTT) were obtained from Genepharma (Shanghai, China). The primary antibodies in this study were used as follows: anti-EGFP (MBL, Nagoya, Japan), anti-E-Cadherin (Cell Signaling Technologies, Danvers, MA), anti-N-Cadherin (Cell Signaling Technologies, Danvers, MA), anti- $\beta$-catenin (Cell Signaling Technologies, Danvers, MA), anti-Vimentin (Epitomics, Burlingame, CA), anti-p-ERK (Santa Cruz Biotechnology, Santa Cruz, CA), anti-ERK (Santa Cruz), anti-p-AKT (Cell Signaling Technologies, Danvers, MA), anti-AKT (Cell Signaling Technologies, Danvers, MA), anti-Fibronectin (BD Biosciences, San Jose, CA), anti-Fibulin (Abcam, Cambridge, MA), anti-Tubulin (Sigma-Aldrich, St.Louis, MO), anti-Cab45 (OriGene Technologies, Rockville, MD), anti-MMP-2 (Epitomics, Burlingame, CA) and anti-GAPDH (Santa Cruz Biotechnology, Santa Cruz, CA). In addition, HRP-conjugated goat anti-mouse/rabbit IgG was purchased from Invitrogen (Carlsbad, CA).

\section{RNA extraction and Real-time PCR}

Total RNA from esophageal tissues was extracted with Trizol (Invitrogen, Carlsbad, CA) and reversely transcribed to cDNA using an oligo(dT) $)_{12}$ primer and Superscript II (Invitrogen). SYBR green dye (Takara Bio Inc., Shiga, Japan) was used for amplification of cDNA. mRNA levels of target genes and the internal standard glyceraldehyde 3-phosphate dehydrogenase (GAPDH) were measured by real-time quantitative PCR (qRT-PCR) in triplicate on a Prism 7900 real-time PCR machine (Applied Biosystems, Foster City, CA). The primers for the transcripts were listed in Table 1.

Table 1. Primer sequences for real-time PCR analysis.

\begin{tabular}{lll}
\hline Gene & Forward primer & Reverse primer \\
\hline GAPDH & $5^{\prime}$-GAAGGTGAAGGTCGGAGTC-3' & $5^{\prime}$-GAAGATGGTGATGGGATTTC-3' \\
MMP2 & $5^{\prime}$-TGATCTTGACCAGAATACCATCGA-3' & $5^{\prime}$-GGCTTGCGAGGGAAGAAGTT-3' \\
MMP7 & $5^{\prime}$-GTGGTCACCTACAGGATCGTA-3' & $5^{\prime}$-CTGAAGTTTCTATTTCTTTCTTGA -3' \\
MMP9 & $5^{\prime}$-GTGCTGGGCTGCTGCTTTGCTG-3' & $5^{\prime}$-GTCGCCCTCAAAGGTTGGAAT-3' \\
MMP13 & 5'-AAGGAGCATGGCGACTTCT -3' & $5^{\prime}$-AAGGAGCATGGCGACTTCT-3' \\
\hline
\end{tabular}




\section{Cell Culture and Transfection}

HeLa, B16, MCF7, MDA231, TE-1 and HEK-293T cells were maintained in DMEM supplemented with 10\% FBS and antibiotics (100 units/ml penicillin G, 100 units/ml streptomycin sulfate; Gibco, Grand Island, NY). Cells were grown in a $37^{\circ} \mathrm{C}$ incubator with $5 \% \mathrm{CO}_{2}$.

For transfection, the cells were grown to $70-80 \%$ confluence and then transfected with vectors using Lipofectamine 2000 (Invitrogen) according to the manufacturer's instructions.

\section{Cab45-G Detection by Enzyme-linked Immunosorbent Assay (ELISA)}

Cab45-G protein in the medium of culture cells was measured using a commercial human Cab45-G ELISA kit (Bluegene, Shanghai, China). The Cab45-G concentration in the medium was calculated using a standard curve obtained from measuring the absorbance of known concentrations of a highly purified recombinant human Cab45-G and expressed in $\mathrm{pg} / \mathrm{ml}$. Briefly, the culture medium was centrifuged at $12000 \times \mathrm{g}$ for $10 \mathrm{~min}$ at $4^{\circ} \mathrm{C}$ and the supernatant was stored at $-80^{\circ} \mathrm{C}$. Thawed supernatant $(100 \mu \mathrm{l})$ was used in the ELISA according to the manufacturer's instructions. A serial dilution of human recombinant Cab45-G was included in each assay to obtain a standard curve from which the sample concentration of Cab45-G could be calculated in $\mathrm{pg} / \mathrm{ml}$ using the measured absorbance values.

\section{Western Blotting}

Cells were transfected with the Cab45-G overexpression vector or shRNAs. Then the cell medium was collected and the cells were washed with ice-cold PBS and collected. All the samples were subject to SDS-PAGE to isolate different proteins. The gel was transferred to a PVDF membrane followed by washing with PBS for three times. Following being blocked with $5 \%$ non-fat milk, the membrane was incubated with the indicated primary antibody at $37^{\circ} \mathrm{C}$ for $1 \mathrm{~h}$. Membrane was washed three times and incubated with peroxidase-conjugated mouse or rabbit secondary antibody for $2 \mathrm{~h}$ at room temperature. Membrane was then washed in TBST, and the detection was done using enhanced chemiluminescence (Beyotime, Nantong, China).

\section{Millicell Assay}

HEK-293T or cancer cells were cultured in the lower chamber and transfected with Cab45-G overexpression or silencing vectors. After $24 \mathrm{~h}$, HeLa or TE-1 cells were plated in the upper chamber. $16 \mathrm{~h}$ later, HeLa or TE-1 cells migrated to the bottom chamber were stained with hematoxylin and quantified manually.

\section{Tissue Samples}

Twenty cervical cancer tissues were obtained from surgical resections of patients between 2011-2012 at Changzhou Tumor Hospital, Soochow University (Changzhou, China). Sixty esophageal cancer tissues were obtained from surgical resections of patients between 2010-2012 at the Changzhou Tumor Hospital (Changzhou, China) and Gastrointestinal Center, Jiangyin People's Hospital (Jiangyin, China), as previously reported [40, 41]. Tissues were obtained before chemotherapy and radiation therapy. All diagnoses were based on pathological and/or cytological evidence. All patients gave signed, informed consent for their tissues to be used for scientific research. Ethical approval for the study was obtained from the Changzhou Tumor Hospital and Jiangyin People's Hospital.

\section{Immunohistochemistry (IHC)}

IHC staining and scoring were performed as reported previously $[40,41]$. In brief, tissues were fixed in $10 \%$ neutral buffered formalin and later embedded in paraffin. Three micrometers thick paraffin sections were deparaffinized and heat treated with citrate buffer, $\mathrm{pH}$ 6.0, for $7 \mathrm{~min}$ as an epitope retrieval protocol. Endogenous peroxidase was blocked with $3 \%$ hydrogen peroxide for $15 \mathrm{~min}$ at room temperature, and tissue non-specific-binding sites were blocked with skimmed milk powder at $4 \%$ applied for $30 \mathrm{~min}$. Sections were then incubated with the Cab45 and MMP-2 antibodies for $1 \mathrm{~h}$ (dilution 1:200) and mixed with skimmed milk powder at $2 \%$ again to reduce unspecific staining. Biotinylated secondary antibody was then added for $30 \mathrm{~min}$. Avidin-biotin-peroxidase complex (Beyotime, Nantong, China) was added and color was developed using 3-3'-diaminobenzidine. Counterstaining was done with hematoxylin. All steps were performed at room temperature.

The criteria for scoring the stained sections were as follows as reported previously $[40,41]$ : negative $(-)$, $<10 \%$ of the whole tissue mass stained; weakly positive $(+1), 10-25 \%$ of the whole tissue mass stained; moderately positive $(+2), 25-75 \%$ of the whole tissue mass stained; and strongly positive $(+3)$, $>75 \%$ of the tissue showed positive.

\section{Statistical Analyses}

Data were expressed as the means \pm SEM of at least three independent experiments. Statistical differences between the test and control values were analyzed by means of Student's $t$-test and Kruskal-Wallis one-way analysis. Correlation analysis between IHC staining score of Cab45-G and MMP-2 
was performed using Spearman's rho test. The quantification of bands was performed using ImageJ software (NIH, Bethesda, MD). Statistical analysis was performed using SPSS software (Release 17.0, SPSS Inc.). Data were considered significant if the two-sided $P<0.05$.

\section{Results}

\section{Cab45-G Is Highly Expressed in Cell Lines with High Metastatic Potential}

We first examined the localization of Cab45-G in HeLa cells. EGFP tag was inserted immediately upstream of Cab45-G and after the signal peptide (SP). We observed that Cab45-G was mainly localized in the Golgi complex as reported previously [10], unlike another CREC family member RCN1, which was localized in endoplastic reticulum (ER) (Fig. 1A). We then tested the secretion of Cab45-G under physiological conditions. As shown in Fig. 1B,
Cab45-G could be detected in the cell lysate and culture medium of Hela cells by Western blot. After transfection of SP-EGFP-Cab45G, Cab45-G was detected more in the culture medium than in total cell lysate (Fig. 5A and 1C), indicative of a role in extracellular space. Given the involvement of some CREC family proteins in cancers, we then investigated the endogenous levels of Cab45-G in different cell lines: B16F10 and MDA-MB-231 with high metastatic potential, while B16F0 and MCF-7 with low metastatic potential. Quantitative real time PCR (qRT-PCR) assay showed that Cab45-G exhibited significantly higher level in B16F10 and MDA-MB-231 than in B16F0 and MCF-7 (Fig. 1D). Results from ELISA confirmed higher Cab45-G level in B16F10 and MDA-MB-231 than in B16F0 and MCF-7 cells (Fig. 1E). These results indicate the association of Cab45-G with cell metastasis.

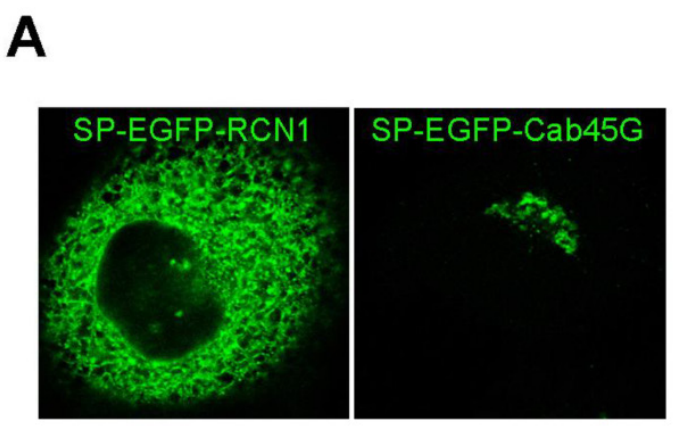

B
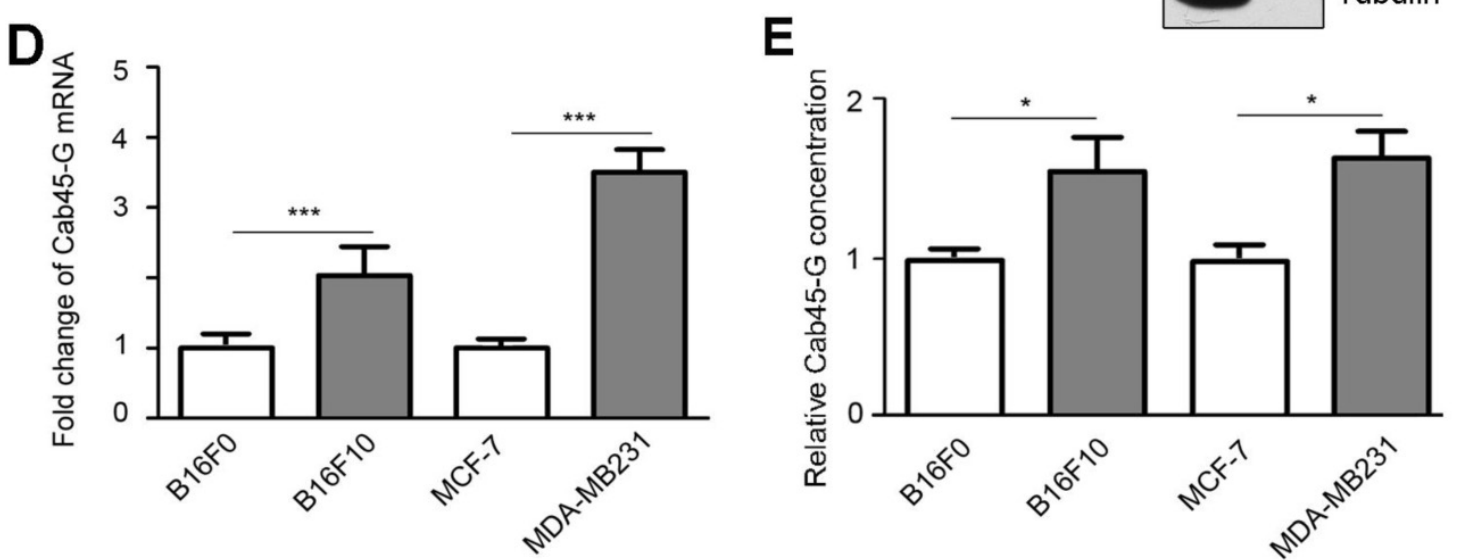

Figure 1. The expression and distribution of Cab45-G in cancer cells. A, HeLa cells were transfected with SP-EGFP-Cab45-G or SP-EGFP-RCN1. The distribution of Cab45-G and RCN1 was observed by a confocal microscope. Overexpressed Cab45-G was localized at Golgi complex in HeLa cells, while its CREC family member RCN1 was localized at endoplasmic reticulum (ER). B, Total cell lysates and cell media from HeLa cells were collected. The expression of Cab45-G and the internal standard Tubulin was analyzed by Western blot. C. Total cell lysates and cell media from HeLa cells with overexpression of SP-EGFP-Cab45-G were analyzed by Western blot. EGFP-tag was inserted after the signal peptide (SP). D, qRT-PCR examination of the endogenous level of Cab45-G in B16F0/F10, MCF-7/MDA-MB-231 cells (the latter cell lines were of higher metastatic potential; $n=3)$. The data are presented as the means \pm SEM and were normalized relative to the control cells. $* * * P<0.001$. E, Conditioned medium from B16F0/F10, MCF-7/MDA-MB-231 cells was collected and assayed for Cab45-G protein by ELISA. Data are presented as means \pm SEM. $* P<0.05$. 


\section{Cab45-G Promotes the Expression of EMT-related Proteins}

We next investigated the effect of Cab45-G on epithelial-mesenchymal transition (EMT), which is a critical step in the tumor metastasis. B16F0 cells transfected with SP-EGFP (control) accumulated together with more cell-cell contacts, while cells transfected with SP-EGFP-Cab45-G were well scattered in a fibroblast-like morphology throughout the space (Fig. 2A). The changing cell morphology and ability to migrate prompted us to test the possibility of endothelial-mesenchymal transition (EMT) upon Cab45-G overexpression. Hence, we detected some marker proteins related to EMT. Western blot analysis showed that overexpression of SP-EGFP-Cab45-G in MCF-7 cells increased the levels of three EMT-related proteins N-Cadherin, $\beta$-Catenin and Vimentin, while decreased the level of E-Cadherin (Fig. 2B). Conversely, knock-down of Cab45-G inhibited the EMT process (Fig. 2C). Taken together, these results suggest a novel function for Cab45-G in the promotion of EMT.

\section{Cab45-G Positively Regulates Cell Migration}

Next, we used Millicell assay to study the role of Cab45-G in cell migration. HEK-293T cells transfected with the Cab45-G overexpression vector (SP-EGFP-Cab45-G) secreted $\sim 1.88$-fold more Cab45-G compared to control vector-transfected (SP-EGFP) cells (Fig. 3A). HEK-293T cells transfected with SP-EGFP (control) or SP-EGFP-Cab45-G were then plated in the lower chamber and HeLa cells were plated in the upper chamber. After 16 hours, HeLa cells migrated to the bottom surface of the membrane were stained with hemotoxilin and calculated manually. Statistical analysis showed that conditioned medium from Cab45-G overexpression HEK-293T cells strikingly promoted the migration of HeLa cells (Fig. 3B). In addition, forced expression of Cab45-G also increased the metastasis of esophageal cancer-derived TE-1 cells (Fig. 3C). To further test the role of Cab45-G in cancer cell metastasis, Cab45-G was overexpressed in Hela (Fig. 3D) and TE-1 cells (Fig. 3F). Results revealed a significant increase in cell migration (Hela and TE-1) by co-culture with the Cab45-G overexpression cancer cells (Fig. 3E and 3G).
A

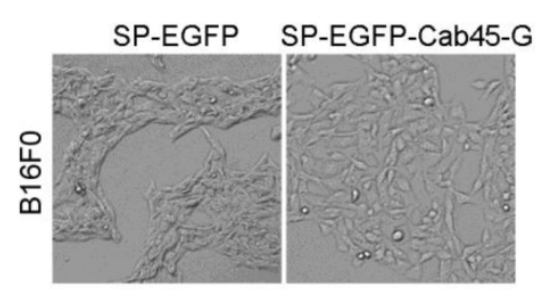

B

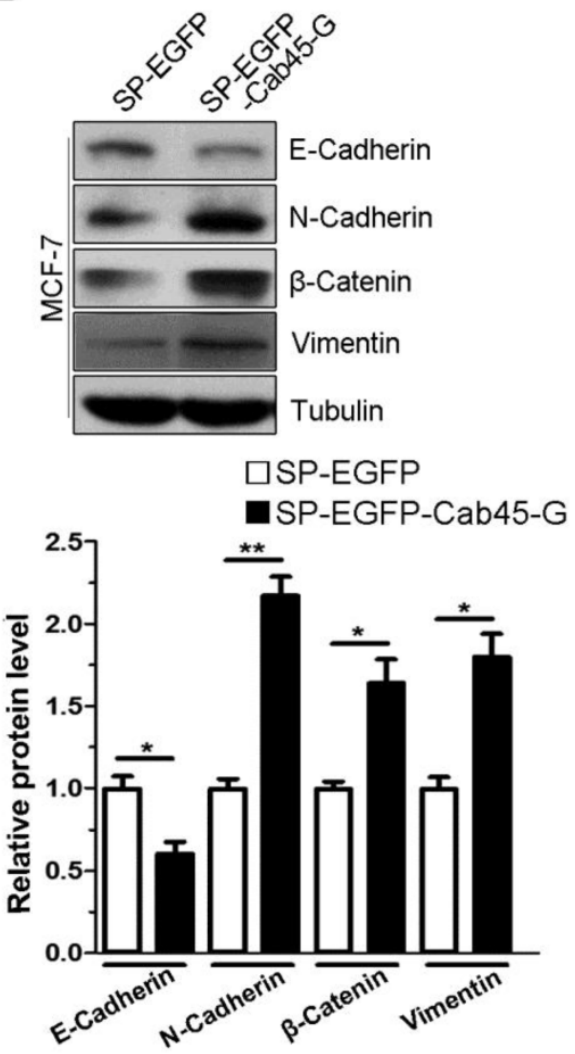

C
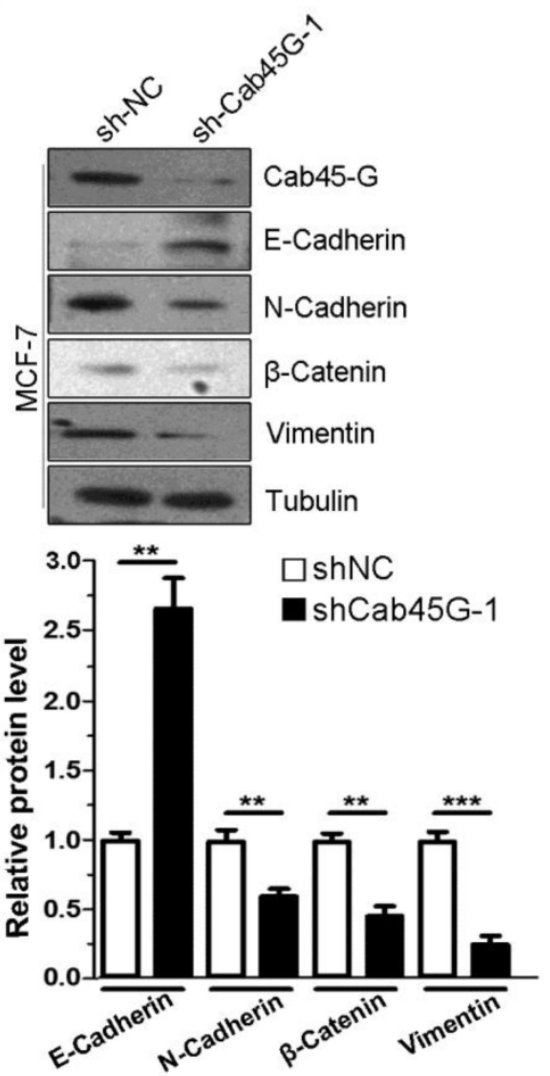

Figure 2. Cab45-G overexpression induced epithelial-mesenchymal transition (EMT) in cancer cells. A, B16F0 cells were transfected with SP-EGFP or SP-EGFP-Cab45-G. The morphology of B16F0 cells was observed by a microscope. B, MCF-7 cells were transfected with SP-EGFP or SP-EGFP-Cab45-G. The expression levels of E-Cadherin, N-Cadherin, $\beta$-Catenin, Vimentin and the internal control Tubulin were determined by Western blot analysis. Relative protein levels were calculated by a SMARTView image analysis system from the Western blot results. C, MCF-7 cells were transfected with shRNA control (sh-NC) or shRNA targeting Cab45-G (sh-Cab45G-1). The expression levels of EMT-related proteins were determined by Western blot analysis. 
A

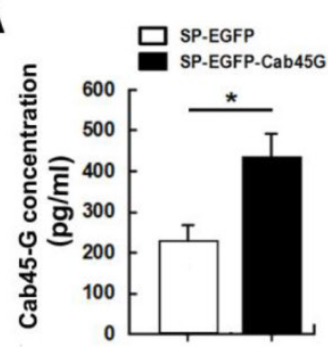

B

SP-EGFP SP-EGFP-Cab45-G

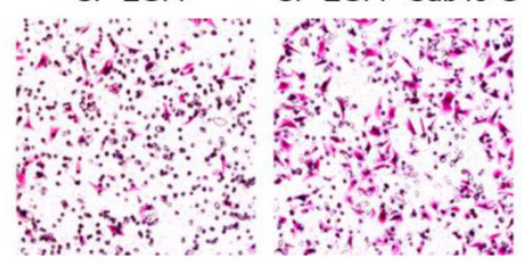

E

SP-EGFP

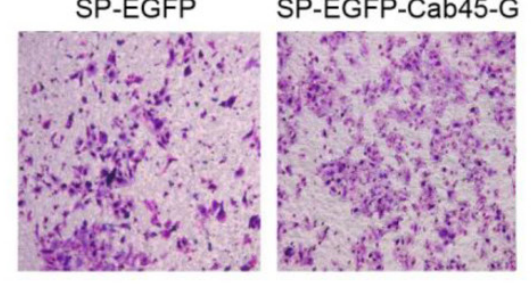

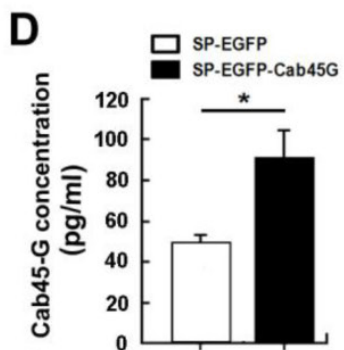

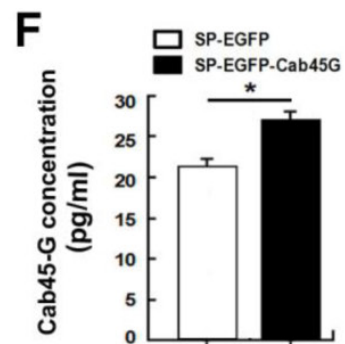

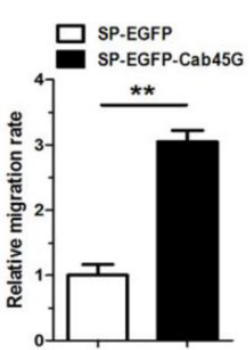

C
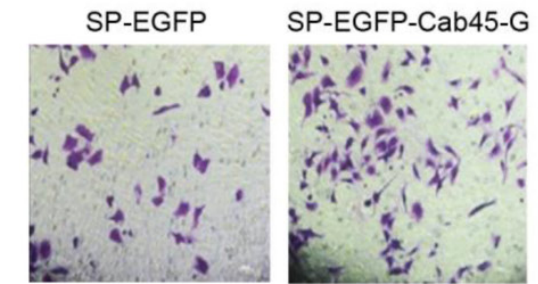

G

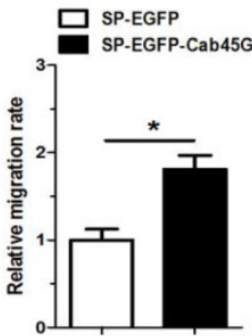

SP-EGFP

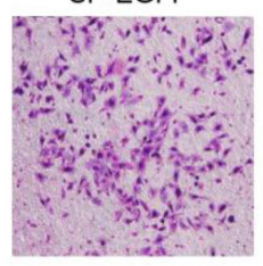

SP-EGFP-Cab45-G

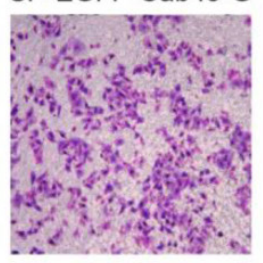

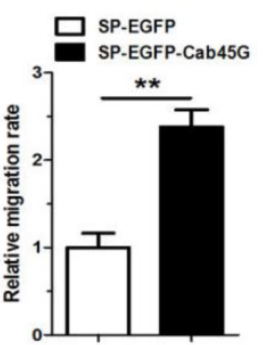

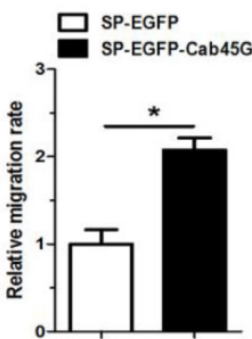

Figure 3. Cab45-G promoted the migration of cancer cells. A, Conditioned medium from SP-EGFP- or SP-EGFP-Cab45-G-transfected HEK-293T cells was collected and assayed for Cab45-G protein by ELISA. Data are presented as means \pm SEM (pg/ml). $* P<0.05$, compared with SP-EGFP-transfected cells. B, Millicell assay of the migration of HeLa cells with SP-EGFP or SP-EGFP-Cab45-G introducing into HEK-293T cells in the lower chamber. After incubation for $16 \mathrm{~h}$, Hela cells migrated to the bottom chamber were stained with hematoxylin. The number of migrated cells was calculated manually. Error bars indicate means $\pm S E M$. $* * P<0.01$. C, Millicell assay of the migration of TE-1 cells with SP-EGFP or SP-EGFP-Cab45-G introducing into HEK-293T cells in the lower chamber. After incubation for $16 \mathrm{~h}$, TE-1 cells migrated to the bottom chamber were stained with hematoxylin. D, Conditioned medium from SP-EGFP- or SP-EGFP-Cab45-G-transfected Hela cells was collected and assayed for Cab45-G protein by ELISA. *P < 0.05. E, Millicell assay of the migration of HeLa cells with SP-EGFP or SP-EGFP-Cab45-G introducing into Hela cells in the lower chamber. Error bars indicate means \pm SEM. $* P<0.05$. F, Conditioned medium from SP-EGFP- or SP-EGFP-Cab45-G-transfected TE- 1 cells was collected and assayed for Cab45-G protein level by ELISA. *P < 0.05 . G, Millicell assay of the migration of TE-1 cells with SP-EGFP or SP-EGFP-Cab45-G introducing into TE-1 cells in the lower chamber. After incubation for 16 h, TE-1 cells migrated to the bottom chamber were stained with hematoxylin. Error bars indicate means \pm SEM. $* P<0.05$.

We then set out to knock down the endogenous expression of Cab45-G to observe its effect on cell migration. Two shRNA sequences against Cab45-G (named sh-Cab45G-1 and sh-Cab45G-2) were designed and confirmed silencing effectiveness in Cab45-G-EGFP stable cell line (Fig. 4A). Similarly, the results obtained from the Western blot and ELISA demonstrated that cells transfected with shCab45G-1 and shCab45G-2 both exhibited $\sim 50 \%$ reduction of the intracellular and extracellular Cab45-G level of the control cells (Fig. 4B and 4C). We then transfected the two shRNAs into HEK-293T cells and performed Millicell assay. The results showed that depletion of Cab45-G by either shCab45G-1 or shCab45G-2 in HEK-293T cells remarkably inhibited the migration of HeLa cells (Fig. 4D). Consistent results were obtained in esophageal cancer-derived TE-1 cells (Fig. 4E). Moreover, silencing of Cab45-G in Hela and TE-1 cells in the lower chamber also suppressed the migration of co-cultured Hela (Fig. 4F and 4G) and TE-1 cells (Fig.
$4 \mathrm{H}$ and $4 \mathrm{I})$, respectively. The above data suggest that Cab45-G serves as a positive factor implicated in tumor cell migration.

\section{Cab45-G Promotes MMP-2 Expression and Activation of ERK Signaling Pathway}

The positive role of Cab45-G in regulating tumor cell migration promoted us to explore the underlying molecular mechanism. The extracellular matrix (ECM) proteins are reported to be important in cell migration and invasion [18], and metalloproteinases (MMPs) regulate the turnover of extracellular matrix by degrading them [19]. Therefore, we investigated the relative mRNA levels of several key ECM proteins and MMPs upon Cab45-G overexpression (Fig. 5A). qRT-PCR assay showed that the expression of several MMPs increased in the mRNA level with the most significant increase for MMP-2 (Fig. 5B). Conversely, knock-down of Cab45-G significantly downregulated the expression of MMP-2 and MMP-7, but not other 
MMPs (Fig. 5D and 5E). These results suggests a role of Cab45-G in regulation of MMP-2 expression and the resultant cell migration.

In response to extracellular signals, ERK signaling functions crucially in MMP expression and tumor cell migration [20]. We then explored the effect of Cab45-G expression on ERK phosphorylation. Western blot analysis showed that SP-EGFP-Cab45-G overexpression in HeLa cells resulted in a marked increase in ERK phosphorylation, but no obvious difference in AKT phosphorylation (Fig. 5C). This result indicates that Cab45-G is specifically involved in the activation of ERK signaling pathway.

\section{Overexpression of Cab45-G Altered the Secretion of ECM Proteins Fibronectin and Fibulin}

In cancer cells, the extracellular matrix (ECM) proteins could supply proinfammatory signals associated with metastasis, invasion and migration [21, 22]. Among ECM proteins, fibulin-1 was reported to interact with fibronectin and inhibit cell migration by disrupting the association between fibronectin and syndecan-4, which played positive roles in cell migration [23]. Considering the characteristic of Cab45-G secretion to cell medium, we asked whether it could affect the secretion of ECM proteins. Consistent with our hypothesis, we observed thatCab45-G overexpression led to a three-fold increase of fibronectin expression in HeLa cells (Fig. $6 \mathrm{~A}$ and $6 \mathrm{~B}$ ), while a $70 \%$ decrease of fibulin secreted to cell medium in comparison with control (Fig. 6A and $6 C)$.
A

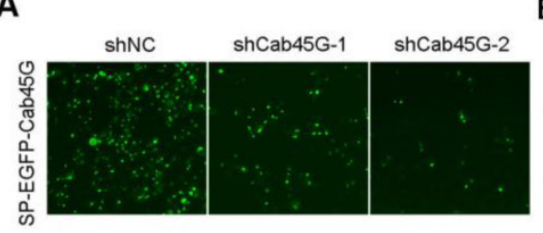

D

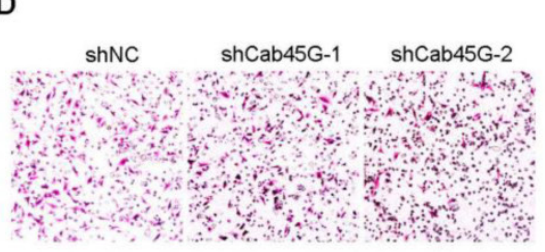

G

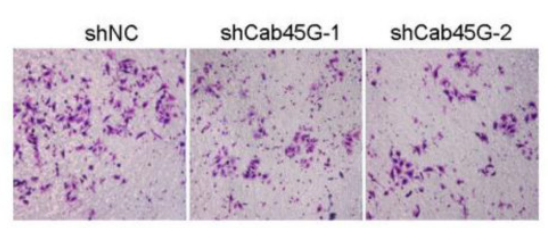

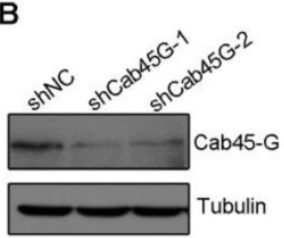
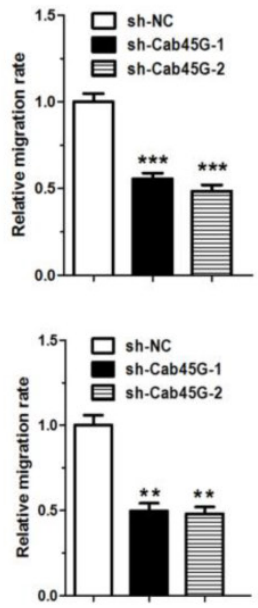

C

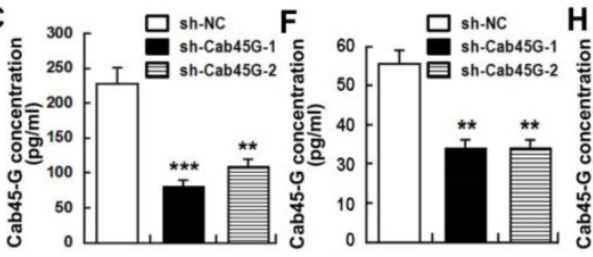

E

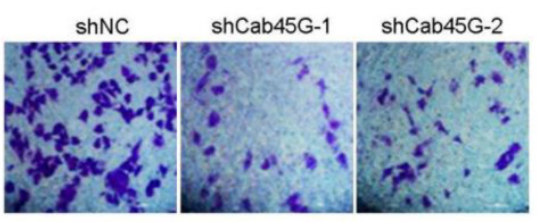

I

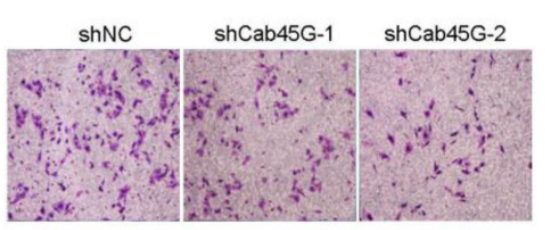

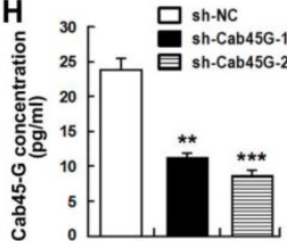
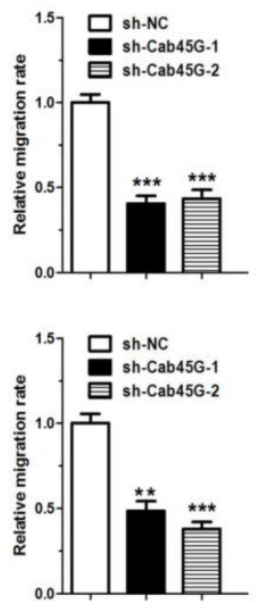

Figure 4. Knock-down of Cab45-G inhibited the migration of cancer cells. A, Cells were transfected with SP-EGFP-Cab45-G in combination with shNA, shCab45G-1 or shCab45G-2. After incubation for 3 days, the efficiency of shRNA on SP-EGFP-Cab45G-transfected HEK-293T cells was observed by a fluorescence microscope. B, Western blot analysis of Cab45-G protein expression after transfection with shNA, shCab45G-1 or shCab45G-2. C, Conditioned medium from shNC-, shCab45G-1- and shCab45G-2-transfected cells was collected and assayed for Cab45-G protein by ELISA. Data are presented as means \pm SEM (pg/ml). $* * P<0.01$; $* * * P<0.001$, compared with shNC-transfected cells. D, Millicell assay of the migration of HeLa cells with shNC, shCab45G-1 or shCab45G-2 introducing into HEK-293T cells in the lower chamber. After incubation for $16 \mathrm{~h}$, Hela cells migrated to the bottom chamber were stained with hematoxylin. The number of migrated Hela cells was calculated manually. The data are presented as the means \pm SEM and were normalized relative to the control cells. $* * * P<0.001$. E, Millicell assay of the migration of TE- 1 cells with shNC, shCab45G- 1 or shCab45G-2 introducing into HEK-293T cells in the lower chamber. After incubation for $16 \mathrm{~h}, \mathrm{TE}-1$ cells migrated to the bottom chamber were stained with hematoxylin. The data are presented as the means \pm SEM and were normalized relative to the control cells. *** $P<0.001$. F, Conditioned medium from shNC-, shCab45G-1- or shCab45G-2-transfected Hela cells was collected and assayed for Cab45-G protein level by ELISA. ** $P<0.01$, compared with shNC-transfected cells. G, Millicell assay of the migration of Hela cells with shNC, shCab45G-1 or shCab45G-2 introducing into Hela cells in the lower chamber. Error bars indicate means \pm SEM. ** $P<0.01$, compared with shNC-transfected cells. H, Conditioned medium from shNC-, shCab45G-1- or shCab45G-2-transfected TE-1 cells was collected and assayed for Cab45-G protein level by ELISA. ** $P<0.01$, *** $P<0.001$, compared with shNC-transfected cells. I, Millicell assay of the migration of TE-1 cells with shNC, shCab45G-1 or shCab45G-2 introducing into TE-1 cells in the lower chamber. Error bars indicate means \pm SEM. $* * P<0.01$; *** $P<0.001$, compared with shNC-transfected cells. 
A

Figure 5. Cab45-G promotes MMPs expression and activation of ERK signaling pathway. A, Hela cells were transfected with SP-EGFP or SP-EGFP-Cab45-G. The protein levels of endogenous and exogenous Cab45-G were determined by Western blot analysis. B, qRT-PCR analysis of the endogenous level of metastasis-related mRNAs in SP-EGFP and SP-EGFP-Cab45G overexpressing HeLa cells $(n=3)$. The data are presented as the means \pm SEM and were normalized relative to the control cells. $* * P<0.01$ $* * * P<0.001$. NS, non-significant. C Western blot analysis of ERK and AKT signaling pathway in HeLa cells transfected with SP-EGFP or SP-EGFP-Cab45-G. D, Hela cells were transfected with shNC or shRNA targeting Cab45-G (shCab45G-1). The protein levels of Cab45-G were determined by Western blot analysis. E, qRT-PCR analysis of the endogenous level of metastasis-related mRNAs in shNC- or shCab45G-1-transfected HeLa cells ( $\mathrm{n}=$ 3 ). The data are presented as the means \pm SEM and were normalized relative to the control cells. $* P<0.05 ; * * * P<0.001$. NS, non-significant.
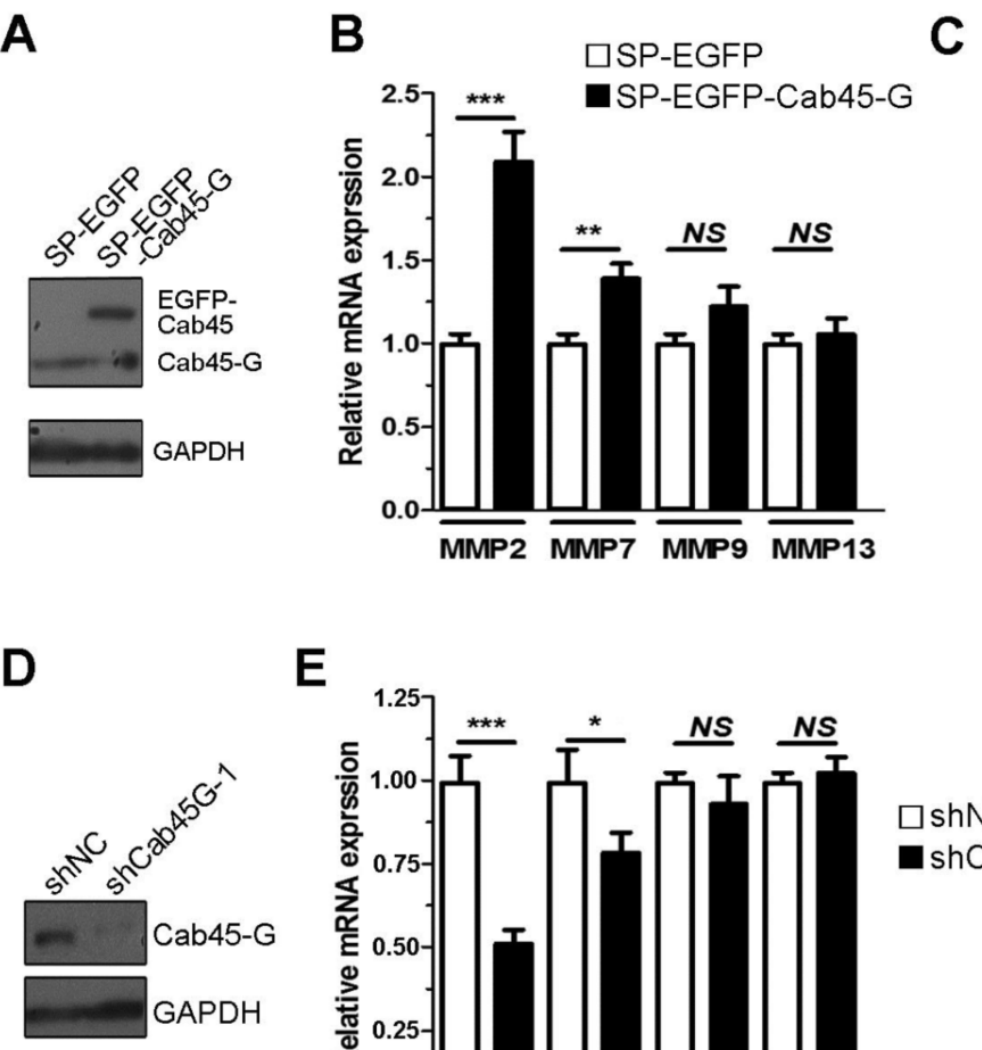

E

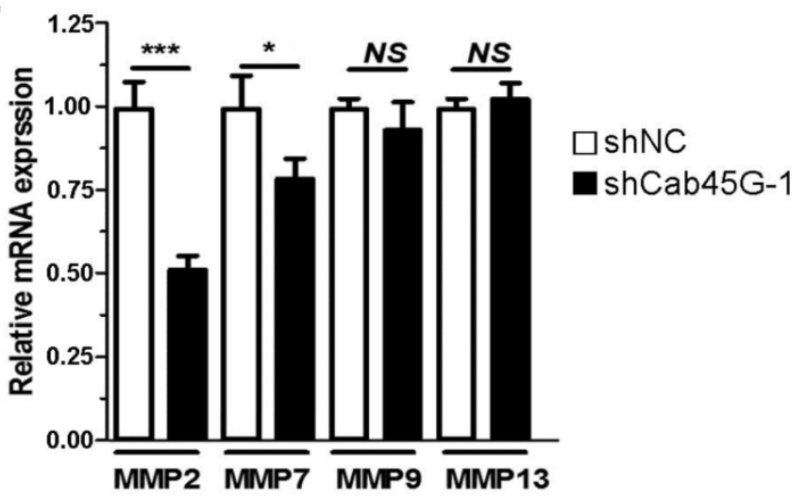

A

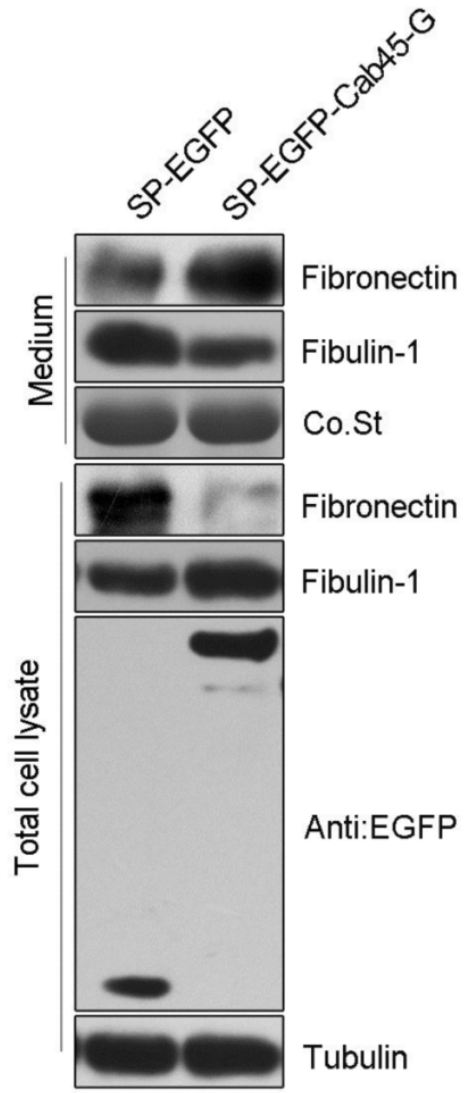

B

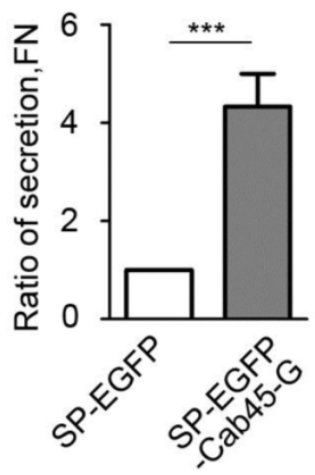

C

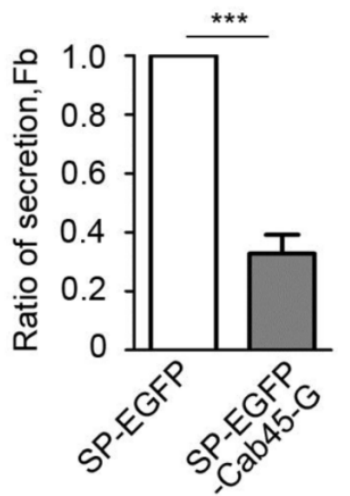

Figure 6. Overexpression of Cab45-G altered the secretion of ECM proteins. A, Hela cells were transfected with SP-EGFP or SP-EGFP-Cab45-G. The protein levels in the cell lysate and culture medium were determined by Western blot analysis. In SP-EGFP-Cab45G overexpressing HeLa cells, secretion of fibronectin (promote metastasis) to cell medium was increased, while fibulin-1 (inhibit metastasis) decreased. B, Quantification of the ratio of fibronectin secretion. The relative mRNA and protein expression levels were analyzed using the Image) image analysis software $(\mathrm{NIH}$, Bethesda, MD). The data are presented as the means \pm SEM and were normalized relative to the control cells. $* * P<0.01$. C, Quantification of the ratio of fibulin secretion. The relative mRNA and protein expression levels were analyzed using the ImageJ image analysis software $(\mathrm{NIH}$, Bethesda, MD). The data are presented as the means \pm SEM and were normalized relative to the control cells. $* * P<0.01$. 


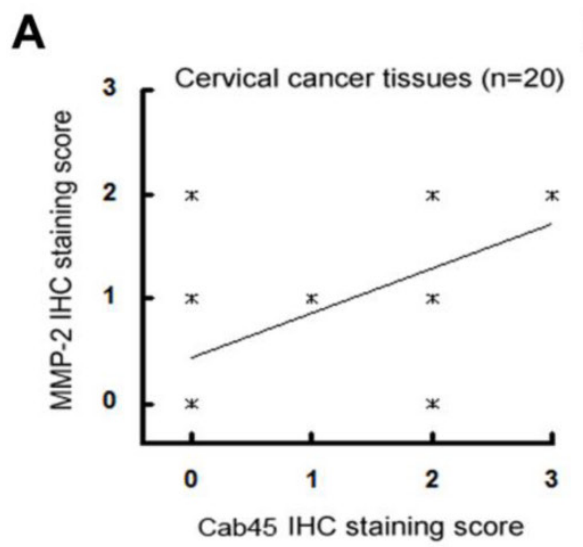

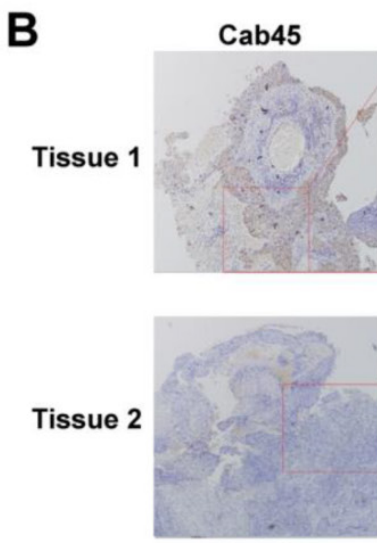

Cab45
Cab45
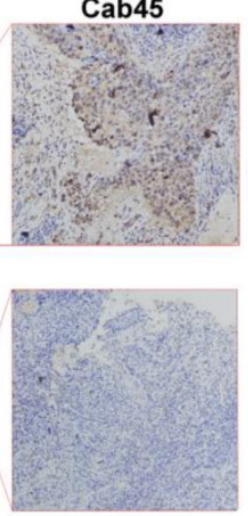

Cab45
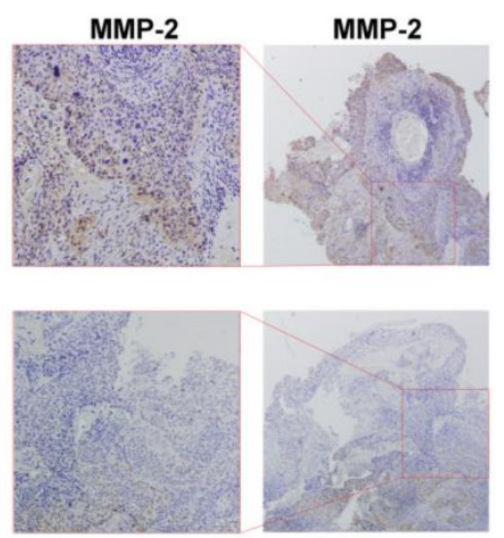

MMP-2

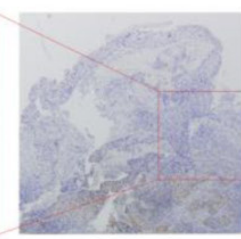

MMP-2

Figure 7. Immunohistochemistry of Cab45 and MMP-2 expression in cervical cancer tissue samples. (A) IHC staining of Cab45 protein showed a significant positive correlation with MMP-2 expression in 20 cervical cancer tissues. (B) Representative IHC staining of both Cab45 and MMP-2 in the same tissue.

A

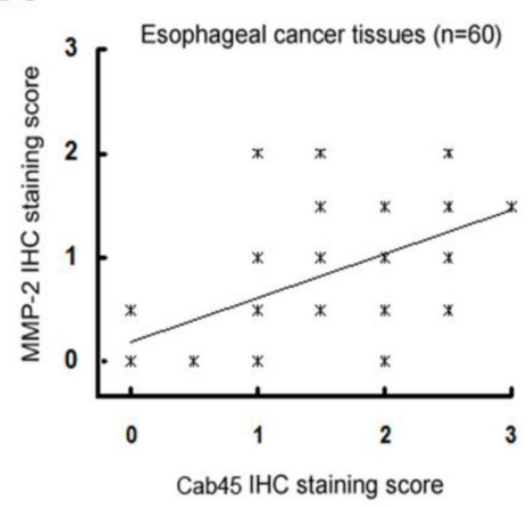

B

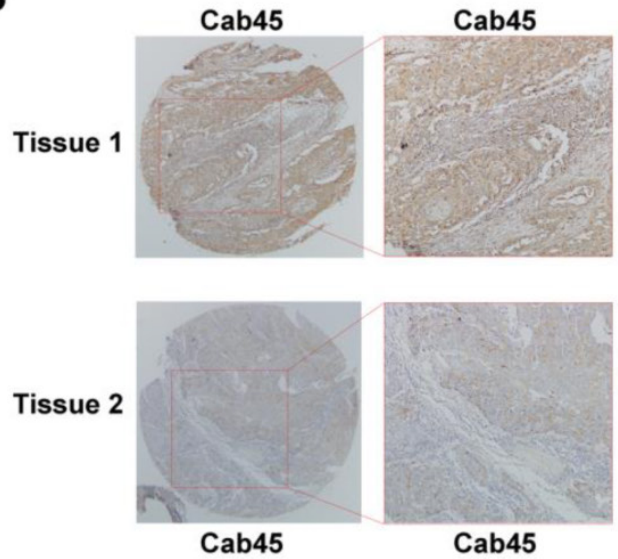

MMP-2
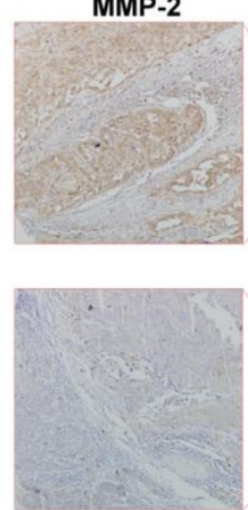

MMP-2
MMP-2
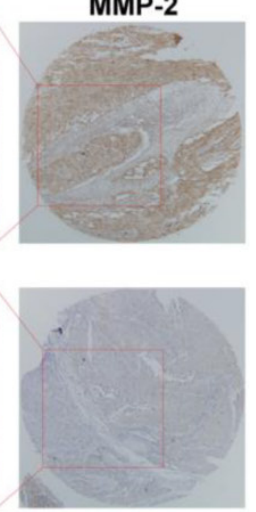

MMP-2

Figure 8. Immunohistochemistry of Cab45 and MMP-2 expression in esophageal cancer tissues. (A) IHC staining of Cab45 protein showed a significant positive correlation with MMP-2 expression in 60 esophageal cancer tissues. (B) Representative IHC staining of both Cab45 and MMP-2 in the same esophageal cancer tissue.

\section{The expression of Cab45-G Correlates with that of MMP-2 in Cancer Tissues}

Because MMP-2 is implicated in cancer metastasis [24-30], to investigate whether Cab45-G expression is associated with that of MMP-2 in human cancer tissues, we analyzed expression of these proteins in human cancer tissue samples (20 cases of cervical cancer tissues and 60 cases of esophageal cancer tissues). Immunohistochemistry analysis showed that, in all cancer tissues examined, expression of Cab45-G is significantly correlated to MMP-2 expression levels (rho $=0.485, P=0.035$ in 20 cervical cancer tissues, Figure 7A; rho $=0.509, P<$ 0.0001 in 60 esophageal cancer tissues, Figure 8A). Representitive images showed that tissue samples with stronger Cab45 staining exhibited increased MMP-2 expression (Figure 7B and 8B). These results indicate that the expression of Cab45 correlates with the expression of MMP-2 in human cancer tissues.

\section{Discussion}

The process of cancer cell invasion and metastasis is a fundamental biological capability during the progression of tumors [31]. Cab45-G, a member of CREC family proteins, has been previously reported to be a secreted protein [10]. However, its function in cancer progression is largely obscure. In this study, we show that Cab45-G is highly expressed in multiple cancer cell lines with stronger metastatic potential (Fig. 1C and 1D) and positively regulates tumor cell migration (Fig. 3 and Fig. 4). Using human cancer tissues, we found that the expression of Cab45 is significantly correlated with that of MMP-2 (Fig. 7 and Fig. 8). These observations are consistent with the evidence of aberrant expression of a number of CREC family proteins in cancer [4]. Overexpression of Cab45-S has been found in cervical and pancreatic cancer tissues [17, 32]. Cab45 subfamily proteins may orchestrate the 
development of human tumors in response to signals triggering tumorigenesis.

Epithelial-mesenchymal transition (EMT) is a regulatory program that enables epithelial cell to acquire the ability to migrate and disseminate [33]. EMT is regarded as a critical event during embryonic development, tumour metastasis and organ fibrosis. The well-defined features of EMT include loss of epithelial markers (E-cadherin and $\alpha$ - and $\gamma$-catenin), gain of mesenchymal cell markers (vimentin, $\beta$-catenin, and $\mathrm{N}$-cadherin) and the acquisition of migratory and invasive properties [34-38]. In accordance with this notion, we observed that the levels of EMT-related proteins N-Cadherin and $\beta$-Catenin are increased and E-Cadherin level decrease upon ectopic expression of Cab45-G (Fig. 2B). This unravels a novel role of CREC proteins in promoting the process of EMT. These results suggest that Cab45-G might play an important role in aggressiveness via regulating EMT. Nevertheless, the underlying mechanism how Cab45-G positively regulates EMT process remains elusive. Further studies are needed to elucidate this mechanism. The appropriate secretion of extracellular matrix proteins contributes largely to the tumor microenvironment [19]. Fibronectin and fibulin are known extracellular matrix proteins, which are implicated in tumor cell migration and metastasis [34-38]. The positive effect of fibronectin on cell migration is compromised by fibulin binding [39]. Consistent with the opposite role of fibronectin and fibulin, we show that overexpression of Cab45-G promotes the secretion of fibronectin, while inhibits that of fibulin to the culture medium (Fig. 6B). The role of Cab45-G in influencing the secretion of extracellular matrix proteins is conceivable as dysregulation of Cab45-G disrupts protein sorting at trans-Golgi network. Since a fraction of Cab45-G is secreted to cell medium, how extracellular Cab45-G coordinates extracellular matrix proteins to modulate cell migration and metastasis needs to be further investigated.

Recent studies have suggested that the ERK signaling is a promising molecular target to control aberrant MMP-2 and MMP-7 expression, and consequent invasion in the EMT-induced non-small cell lung cancer (NSCLC) cells [42]. Our study showed that Cab45-G overexpression resulted in a marked increase in ERK phosphorylation and MMP-2 mRNA level in HeLa cells. Thus the Cab45G/ERK/MMP2 axis is, at least partially, responsible for promoting invasion in cancer cells. Nevertheless, at present the underlying mechanisms how Cab45-G regulates ERK phosphorylation remains elusive. Since Cab45-G was mainly present in the Golgi apparatus and extracellular space, Cab45-G may exert its function through intracellular and receptor-mediated manners. Further studies are needed to investigate the mechanism of Cab45-G in regulating ERK phosphorylation.

In conclusion, we found that Cab45-G could regulate cancer cell migration through various molecular mechanisms, which may serve as a promising therapeutic target for the treatment of cancers.

\section{Acknowledgements}

This work is supported by the National Natural Science Foundation of China $(81402518,81472920$ and 81522039), Jiangsu Provincial Special Program of Medical Science (BE2015631), Natural Science Foundation of Jiangsu Province (BK20151174) and the Scientific Research Foundation of Third Institute of Oceanography, SOA (2014007).

\section{Competing Interests}

The authors have declared that no competing interest exists.

\section{References}

1. Yabe D, Taniwaki M, Nakamura T, Kanazawa N, Tashiro K and Honjo T. Human calumenin gene (CALU): cDNA isolation and chromosomal mapping to 7 q32. Genomics. 1998; 49:331-333.

2. Strynadka NC and James MN. Crystal structures of the helix-loop-helix calcium-binding proteins. Annu Rev Biochem.1989;58:951-998.

3. Honore B and Vorum $\mathrm{H}$. The CREC family, a novel family of multiple EF-hand, low-affinity $\mathrm{Ca}(2+)$-binding proteins localised to the secretory pathway of mammalian cells. FEBS Lett. 2000;466:11-18.

4. Honore B. The rapidly expanding CREC protein family: members, localization, function, and role in disease. Bioessays. 2009;31:262-277.

5. von Blume J, Alleaume AM, Kienzle C, Carreras-Sureda A, Valverde M and Malhotra V. Cab45 is required for $\mathrm{Ca}(2+)$-dependent secretory cargo sorting at the trans-Golgi network. J Cell Biol. 2012;199:1057-1066.

6. Tong A, Zhang H, Li Z, Gou L, Wang Z, Wei H, et al. Proteomic analysis of liver cancer cells treated with suberonylanilide hydroxamic acid. Cancer Chemother Pharmacol. 2008;61:791-802.

7. Zhu Y, Wang Q, Xu W and Li S. The ethanol response gene Cab45 can modulate the impairment elicited by ethanol and ultraviolet in PC12 cells. J Genet Genomics. 2008;35:153-161.

8. Wang $\mathrm{Q}, \mathrm{Zhu} X, \mathrm{Xu} \mathrm{O}$ Ding $\mathrm{X}$, Chen $\mathrm{YE}$ and Song $\mathrm{Q}$. Effect of C-reactive protein on gene expression in vascular endothelial cells. Am J Physiol Heart Circ Physiol. 2005;288:H1539-1545.

9. Tsuji A, Kikuchi Y, Sato Y, Koide S, Yuasa K, Nagahama M, et al. A proteomic approach reveals transient association of reticulocalbin-3, a novel member of the CREC family, with the precursor of subtilisin-like proprotein convertase, PACE4. Biochem J. 2006;396:51-59.

10. Scherer PE, Lederkremer GZ, Williams S, Fogliano M, Baldini G and Lodish HF. Cab45, a novel (Ca2+)-binding protein localized to the Golgi lumen. J Cell Biol. 1996;133:257-268.

11. Lam PP, Hyvarinen K, Kauppi M, Cosen-Binker L, Laitinen S, Keranen S, et al. A cytosolic splice variant of Cab45 interacts with Munc18b and impacts on amylase secretion by pancreatic acini. Mol Biol Cell. 2007;18: 2473-2480.

12. Lara-Lemus $R$, Liu M, Turner MD, Scherer $P$, Stenbeck $G$, Iyengar $P$, et al. Lumenal protein sorting to the constitutive secretory pathway of a regulated secretory cell. J Cell Sci. 2006;119:1833-1842.

13. Stacker SA, Baldwin ME and Achen MG. The role of tumor lymphangiogenesis in metastatic spread. FASEB J. 2002;16:922-934.

14. Da MX, Wu Z and Tian HW. Tumor lymphangiogenesis and lymphangiogenic growth factors. Arch Med Res. 2008;39:365-372.

15. Meng $\mathrm{F}$ and $\mathrm{Wu} \mathrm{G}$. The rejuvenated scenario of epithelial-mesenchymal transition (EMT) and cancer metastasis. Cancer Metastasis Rev. 2012;31:455-467

16. Samatov TR, Tonevitsky AG and Schumacher U. Epithelial-mesenchymal transition: focus on metastatic cascade, alternative splicing, non-coding RNAs and modulating compounds. Mol Cancer. 2013;12:107.

17. Chen L, Xu S, Xu Y, Lu W, Liu L, Yue D, Teng J, Chen J. Cab45S promotes cell proliferation through SERCA2b inhibition and $\mathrm{Ca} 2+$ signaling. Oncogene. 2015. doi: 10.1038 /onc. 2015.56 . 
18. Hynes RO. The extracellular matrix: not just pretty fibrils. Science. 2009;326:1216-1219.

19. Kessenbrock K, Plaks V and Werb Z. Matrix metalloproteinases: regulators of the tumor microenvironment. Cell. 2010;141:52-67.

20. Huang C, Jacobson $\mathrm{K}$ and Schaller MD. MAP kinases and cell migration. J Cell Sci. 2004;117:4619-4628.

21. Lu P, Weaver VM and Werb Z. The extracellular matrix: a dynamic niche in cancer progression. J Cell Biol. 2012;196:395-406.

22. Gritsenko PG, Ilina $O$ and Friedl P. Interstitial guidance of cancer invasion. J Pathol. 2012;226:185-199.

23. Twal WO, Czirok A, Hegedus B, Knaak C, Chintalapudi MR, Okagawa H, et al. Fibulin-1 suppression of fibronectin-regulated cell adhesion and motility. J Cell Sci. 2001;114:4587-4598.

24. Hajitou A, Sounni NE, Devy L, Grignet-Debrus C, Lewalle JM, Li H, et al. Down-regulation of vascular endothelial growth factor by tissue inhibitor of metalloproteinase-2: effect on in vivo mammary tumor growth and angiogenesis. Cancer Res. 2001;61:3450-3457.

25. Yamasaki M, Nagatomo T, Matsuyama T, Ikeho Y, Kato E, Nishiyama K, et al. Conjugated linoleic acids inhibit hypoxia inducible factor-1alpha stabilization under hypoxic condition in human hepatocellular carcinoma cells. J Oleo Sci. 2012;61:491-496.

26. Birner P, Schindl M, Obermair A, Plank C, Breitenecker G and Oberhuber G. Overexpression of hypoxia-inducible factor 1alpha is a marker for an unfavorable prognosis in early-stage invasive cervical cancer. Cancer Res. 2000;60:4693-4696.

27. Karahan N, Guney M, Baspinar S, Oral B, Kapucuoglu N and Mungan T. Expression of gelatinase (MMP-2 and MMP-9) and cyclooxygenase-2 (COX-2) in endometrial carcinoma. Eur J Gynaecol Oncol. 2007;28:184-188.

28. Sakata H, Fujimura M, Watanabe $M$ and Tominaga T. Association of cavernous malformation within vestibular schwannoma: immunohistochemical analysis of matrix metalloproteinase-2 and -9. Neurol Med Chir (Tokyo). 2007;47:509-512.

29. Du R, Petritsch C, Lu K, Liu P, Haller A, Ganss R, et al. Matrix metalloproteinase-2 regulates vascular patterning and growth affecting tumor cell survival and invasion in GBM. Neuro Oncol. 2008;10: 254-264.

30. Xiang ZL, Zeng ZC, Fan J, Tang ZY, Zeng HY and Gao DM. Gene expression profiling of fixed tissues identified hypoxia-inducible factor-1alpha, VEGF, and matrix metalloproteinase- 2 as biomarkers of lymph node metastasis in hepatocellular carcinoma. Clin Cancer Res. 2011;17:5463-5472.

31. Talmadge JE and Fidler IJ. AACR centennial series: the biology of cancer metastasis: historical perspective. Cancer Res.2010;70:5649-5669.

32. Chen L, Xu S, Liu L, Wen X, Xu Y, Chen J et al. Cab45S inhibits the ER stress-induced IRE1-JNK pathway and apoptosis via GRP78/BiP. Cell Death Dis. 2014;5: e1219.

33. Yilmaz M and Christofori G. EMT, the cytoskeleton, and cancer cell invasion. Cancer Metastasis Rev. 2009;28:15-33.

34. Thiery JP, Acloque H, Huang RY and Nieto MA. Epithelial-mesenchymal transitions in development and disease. Cell. 2009;139:871-890.

35. Yang J and Weinberg RA. Epithelial-mesenchymal transition: at the crossroads of development and tumor metastasis. Dev Cell. 2008;14:818-829.

36. Yang $\mathrm{MH}$ and $\mathrm{Wu} \mathrm{KJ}$. TWIST activation by hypoxia inducible factor-1 (HIF-1): implications in metastasis and development. Cell Cycle. 2008;7:2090-2096.

37. Higgins DF, Kimura K, Bernhardt WM, Shrimanker N, Akai Y, Hohenstein B, et al. Hypoxia promotes fibrogenesis in vivo via HIF-1 stimulation of epithelial-to-mesenchymal transition. J Clin Invest. 2007;117:3810-3820.

38. Thompson EW, Newgreen DF and Tarin D. Carcinoma invasion and metastasis: a role for epithelial-mesenchymal transition? Cancer Res. 2005;65:5991-5995; discussion 5995.

39. Hayashido Y, Lucas A, Rougeot C, Godyna S, Argraves WS and Rochefort H. Estradiol and fibulin-1 inhibit motility of human ovarian- and breast-cancer cells induced by fibronectin. Int J Cancer. 1998;75:654-658.

40. Luo J, Zhou X, Ge X, Liu P, Cao J, Lu X, et al. Upregulation of Ying Yang 1 (YY1) suppresses esophageal squamous cell carcinoma development through heme oxygenase-1. Cancer Sci. 2013;104:1544-1551.

41. Liu P, Chen S, Wu W, Liu B, Shen W, Wang F, et al. Contactin-1 (CNTN-1) overexpression is correlated with advanced clinical stage and lymph node metastasis in oesophageal squamous cell carcinomas. Jpn J Clin Oncol. 2012;42:612-618

42. Bae G, Choi S, Lee J, Jo J, Lee J, Kim J et al. Loss of E-cadherin activates EGFR-MEK/ERK signaling, which promotes invasion via the ZEB1/MMP2 axis in non-small cell lung cancer. Oncotarget 2013;4:2512-2522. 